TRANSACTIONS OF THE

AMERICAN MATHEMATICAL SOCIETY

Volume 349, Number 6, June 1997, Pages 2359-2370

S $0002-9947(97) 01736-4$

\title{
A FINITENESS THEOREM FOR LOW-CODIMENSIONAL NONSINGULAR SUBVARIETIES OF QUADRICS
}

\author{
MARK ANDREA A. DE CATALDO
}

\begin{abstract}
We prove that there are only finitely many families of codimension two nonsingular subvarieties of quadrics $\mathcal{Q}^{n}$ which are not of general type, for $n=5$ and $n \geq 7$. We prove a similar statement also for the case of higher codimension.
\end{abstract}

\section{INTRODUCTION}

There are only finitely many families of codimension two nonsingular subvarieties not of general type of the projective spaces $\mathbb{P}^{n}$, for $n \geq 4$; see [7] and [4]. More generally, a similar statement holds for the case of higher codimension; see [16].

In this paper we concentrate on the case of codimension two subvarieties of quadrics. Our main result is Theorem 4.3: there are only finitely many families of nonsingular codimension two subvarieties not of general type in the quadrics $\mathcal{Q}^{n}$, $n=4,5$ or $n \geq 7$. The case $n=4$ is proved in [1], $\S 6$. The case of $n=5$ is at the heart of the paper; the main tools are the semipositivity of the normal bundles of nonsingular subvarieties of quadrics, the double point formula, the generalized Hodge index theorem, bounds for the genus of curves on $\mathcal{Q}^{3}$, Proposition 1.5 and Corollary 3.4. The case $n=6$ is still open ${ }^{1}$. The case of codimension two with $n \geq 7$ is covered by Theorem 2.1, which hinges upon the result of [16]; it also gives a finiteness result in codimension bigger than two in the same spirit as [16].

The paper is organized as follows. Section 1 records, for the reader's convenience, some results used in the paper. A generalization of a lifting criterion of Roth's is contained in section 1.1; we shall need the particular case expressed by Proposition 1.4. Section 2 deals with higher codimensional cases. Sections 3 and 4 are modeled on [4]. Section 3 contains the lengthy proof of Theorem 3.1 and of its Corollary 3.4. Section 4 contains the proof of Theorem 4.3 .

Notation and conventions. Our basic reference is [10]. We work over any algebraically closed field of characteristic zero. A quadric $\mathcal{Q}^{n}$, here, is a nonsingular hypersurface of degree two in the projective space $\mathbb{P}^{n+1}$. Little or no distinction is made between line bundles, associated sheaves of sections and Cartier divisors; moreover the additive and tensor notation are both used.

\footnotetext{
Received by the editors November 27, 1995.

1991 Mathematics Subject Classification. Primary 14J70, 14M07, 14M10, 14M15, 14M17, $14 \mathrm{M} 20$.

Key words and phrases. Codimension two, Grassmannians, lifting, low codimension, not of general type, polynomial bound, quadrics.

${ }^{1}$ L. Fania and G. Ottaviani have recently informed the author that they have settled the case $n=6$ in the positive.
} 


\section{ACKNOWLEDGMENTS}

It is a pleasure to thank my Ph.D. advisor A.J. Sommese, who suggested that I study 3 -folds in $\mathcal{Q}^{5}$.

\section{Preliminary material}

Proposition 1.1 (Cf. [6] or [5]; for the case of $d>2 k(k-1)$ see [1], $\S 6)$. Let $C$ be an integral curve of degree $d$ contained in an integral surface of degree $2 k$ in $\mathcal{Q}^{3}$. Then the following bound holds for the genus $g$ of $C$ :

$$
g-1 \leq \frac{d^{2}}{4 k}+\frac{1}{2}(k-3) d
$$

Proposition 1.2 (Cf. [1], Proposition 6.4). Let $C$ be an integral curve in $\mathcal{Q}^{3}$, not contained in any surface in $\mathcal{Q}^{3}$ of degree strictly less than $2 k$. Then

$$
g-1 \leq \frac{d^{2}}{2 k}+\frac{1}{2}(k-4) d
$$

Let $S$ be a nonsingular surface in $\mathcal{Q}^{4}, N$ its normal bundle, $C$ a nonsingular hyperplane section of $S, g$ its genus, $d$ its degree. Let $V_{s} \in\left|\mathcal{I}_{S, \mathcal{Q}^{4}}(s)\right|$, where $s$ is some positive integer, be an integral hypersurface and $\mu_{l}:=c_{2}(N(-l))=$ $(1 / 2) d^{2}+l(l-3) d-2 l(g-1), \forall l \in \mathbb{Z}$.

Lemma 1.3 (Cf. [5], Lemma 2.35). In the above situation,

$$
0 \leq \mu_{s} \leq s^{2} d
$$

The following proposition follows immediately from Theorem 1.5 when the ambient space $\mathcal{P}^{n+2}$ is chosen to be a quadric $\mathcal{Q}^{n+2}$.

Proposition 1.4. Let $X$ be an integral subscheme of degree $d$ and codimension two in $\mathcal{Q}^{n}, n \geq 4$. Assume that for the general hyperplane section $Y$ of $X$ we have

$$
h^{0}\left(\mathcal{Q}^{n-1}, \mathcal{I}_{Y, \mathcal{Q}^{n-1}}(\sigma)\right) \neq 0,
$$

for some positive integer $s$ such that $d>2 \sigma^{2}$. Then

$$
h^{0}\left(\mathcal{Q}^{n}, \mathcal{I}_{X, \mathcal{Q}^{n}}(\sigma)\right) \neq 0 .
$$

Let $X$ be a degree $d$, nonsingular 3 -fold in $\mathcal{Q}^{5}, L \simeq \mathcal{O}_{\mathbb{P}^{6}}(1)_{\mid X}, S$ the surface general hyperplane section of $X, C$ the general curve section of $S$ and $g$ the genus of $C$. Using the double point formula (cf. [8]) for the embedding $X \hookrightarrow \mathcal{Q}^{5}$, we get the following formulæ for $K_{X} \cdot L^{2}, K_{X}^{2} \cdot L, K_{X}^{3}$ as functions of $d, g, \chi\left(\mathcal{O}_{X}\right), \chi\left(\mathcal{O}_{S}\right)$ :

$$
\begin{gathered}
K_{X} \cdot L^{2}=2(g-1)-2 d, \\
K_{X}^{2} \cdot L=\frac{1}{4} d^{2}+\frac{3}{2} d-8(g-1)+6 \chi\left(\mathcal{O}_{S}\right), \\
K_{X}^{3}=-\frac{9}{4} d^{2}+\frac{27}{2} d+g d+18(g-1)-30 \chi\left(\mathcal{O}_{S}\right)-24 \chi\left(\mathcal{O}_{X}\right) .
\end{gathered}
$$

Finally we record the expression for the Hilbert polynomial of $X$ :

$$
\chi\left(\mathcal{O}_{X}(t)\right)=\frac{1}{6} d t^{3}+\left[\frac{1}{2} d-\frac{1}{2}(g-1)\right] t^{2}+\left[\frac{1}{3} d-\frac{1}{2}(g-1)+\chi\left(\mathcal{O}_{S}\right)\right] t+\chi\left(\mathcal{O}_{X}\right) .
$$

For the details concerning the above formulæ see [5], §1. 
1.1. A Roth-type lifting criterion. If the general curve section of a degree $d$ linearly normal surface $S$ in $\mathbb{P}^{4}$ lies on a surface of degree $\sigma$ in $\mathbb{P}^{3}$, then $S$ lies on some hypersurface of degree $\sigma$, provided $d>\sigma^{2}$ (cf. [15]). A generalization of this fact to codimension two integral linearly normal subschemes of $\mathbb{P}^{n}, n \geq 4$, has been known for some time.

In this section we generalize Roth's lifting criterion to a larger class of spaces; see Theorem 1.5 and Example 1.8. The proof does not require the concept of linear normality, which was virtually automatic in the case that Roth considered.

The proof given below was inspired by [1], Lemma 6.1.

First we fix some notation. Let $\mathcal{P}^{n+2}$ be a nonsingular projective variety of dimension $(n+2), n \geq 2, L=\mathcal{O}_{\mathcal{P}^{n+2}}(1)$ an ample and spanned line bundle on it with $\delta:=L^{n+2}$. Assume that $\operatorname{Pic}\left(\mathcal{P}^{n+2}\right) \simeq \mathbb{Z}[L]$. Let $X^{n}$ be an integral subscheme of $\mathcal{P}^{n+2}$ of dimension $n$ and $d:=L^{n} \cdot X$. Denote by $\mathcal{P}^{i+2}$ the intersection of $(n-i)$ general elements of $|L|$ and by $X^{i}$ the intersection of the same elements of $|L|$ with $X^{n}$.

Theorem 1.5. Assume that the natural restriction maps below are surjective $\forall m$ :

$$
\rho_{m}:=H^{0}\left(\mathcal{P}^{n+2}, m L\right) \rightarrow H^{0}\left(\mathcal{P}^{n+1}, m L_{\mid \mathcal{P}^{n+1}}\right) .
$$

If $h^{0}\left(\mathcal{I}_{X^{n-1}, \mathcal{P}^{n+1}}(s)\right) \neq 0$ for some $s$ such that $d>\delta s^{2}$, then $h^{0}\left(\mathcal{I}_{X^{n}, \mathcal{P}^{n+2}}(s)\right) \neq 0$.

If $s$ is the minimum such number, then $h^{0}\left(\mathcal{I}_{X^{n}, \mathcal{P}^{n+2}}(s)\right)=1$.

Proof. Let us assume that we have proved the theorem for

$$
s=\sigma:=\min \left\{t \in \mathbb{N} \mid h^{0}\left(\mathcal{I}_{X^{n-1}, \mathcal{P}^{n+1}}(t)\right) \neq 0\right\}
$$

we call $\sigma$ the postulation of $X^{n-1}$. Then the theorem holds also for all $s \geq \sigma$. We can thus assume, without loss of generality, that $s=\sigma$.

Pick any $V_{\sigma} \in\left|\mathcal{I}_{X^{n-1}, \mathcal{P}^{n+1}}(\sigma)\right|$.

Claim. $V_{\sigma}$ is integral. This follows easily from the minimality of $\sigma$ and the fact that, under our assumptions, $\operatorname{Pic}\left(\mathcal{P}^{n+1}\right) \simeq \mathbb{Z}\left[L_{\mid \mathcal{P}^{n+1}}\right]$.

Claim. $V_{\sigma}$ is the unique element of $\left|\mathcal{I}_{X^{n-1}, \mathcal{P}^{n+1}}(\sigma)\right|$. For a contradiction, assume that we have two distinct $V_{\sigma}^{i}$. By the above claim they are both integral. By an easy Bertini-type argument we see that, intersecting everything with $n$ general members of $|L|$, we get two distinct integral curves $W_{\sigma}^{i} \in\left|\mathcal{O}_{\mathcal{P}^{2}}(\sigma)\right|$ containing $X^{0}=\{d$ points $\}$. Since the curves do not have common components, we see that $d \leq W_{\sigma}^{1} \cdot W_{\sigma}^{2}=\delta \sigma^{2}$; the intersection product here is on $\mathcal{P}^{2}$. This is a contradiction, and the claim is proved.

Let us choose a general line $\ell \subseteq|L|^{\vee}$. Define $\tilde{\mathcal{P}}$ to be the blowing up of $\mathcal{P}^{n+2}$ along the intersection of all the members of $\ell$. Denote by $p$ and $q$ the natural projections to $\ell$ and $\mathcal{P}^{n+2}$, respectively. By intersecting with general elements of $|L|$ we get the following diagram, where $Y^{i}$ denotes $q^{-1} X^{i}$ :

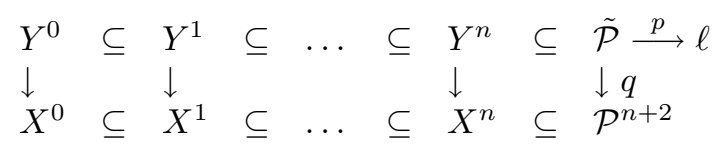

We have the following injections, where, for simplicity (and by abuse) of notation, we denote a twist by $q^{*} \mathcal{O}_{\mathcal{P}^{n+2}}(\sigma)$ simply by a twist by $\sigma$ :

$$
\mathcal{I}_{Y^{n}, \tilde{\mathcal{P}}}(\sigma) \rightarrow \mathcal{I}_{Y^{n-1}, \tilde{\mathcal{P}}}(\sigma) \rightarrow \ldots \rightarrow \mathcal{I}_{Y^{1}, \tilde{\mathcal{P}}}(\sigma) \rightarrow \mathcal{I}_{Y^{0}, \tilde{\mathcal{P}}}(\sigma)
$$


so that, applying $p_{*}$, we obtain the following injections:

$$
p_{*} \mathcal{I}_{Y^{n}, \tilde{\mathcal{P}}}(\sigma) \rightarrow p_{*} \mathcal{I}_{Y^{n-1}, \tilde{\mathcal{P}}}(\sigma) \rightarrow \ldots \rightarrow p_{*} \mathcal{I}_{Y^{1}, \tilde{\mathcal{P}}}(\sigma) \rightarrow p_{*} \mathcal{I}_{Y^{0}, \tilde{\mathcal{P}}}(\sigma)
$$

The existence of $V_{\sigma}$, for a general point of $\ell$, ensures that $p_{*} \mathcal{I}_{Y^{n}, \tilde{\mathcal{P}}}(\sigma)$ is not the zero sheaf. Since $p$ is dominant and the ideal sheaves $\mathcal{I}_{Y^{i}, \tilde{\mathcal{P}}}$ are torsion free, we see that the sheaves $p_{*} \mathcal{I}_{Y^{i}, \tilde{\mathcal{P}}}(\sigma)$ are torsion free $\forall i$. But $\ell$ is a smooth curve, so that these sheaves are actually locally free. The uniqueness statement, which was shown above, implies that these sheaves are actually line bundles on $\ell$. Since each of the above injections has torsion free cokernel on $\ell$ we deduce that they all are isomorphisms, i.e.:

$$
p_{*} \mathcal{I}_{Y^{n}, \tilde{\mathcal{P}}}(\sigma) \simeq p_{*} \mathcal{I}_{Y^{n-1}, \tilde{\mathcal{P}}}(\sigma) \simeq \ldots \simeq p_{*} \mathcal{I}_{Y^{1}, \tilde{\mathcal{P}}}(\sigma) \simeq p_{*} \mathcal{I}_{Y^{0}, \tilde{\mathcal{P}}}(\sigma) \simeq \mathcal{O}_{\ell}(\tau),
$$

for some $\tau \in \mathbb{Z}$.

For a contradiction, assume $\tau<0$. Then

$0=h^{0}\left(p_{*} \mathcal{I}_{Y^{n-1}, \tilde{\mathcal{P}}}(\sigma)\right)=h^{0}\left(\mathcal{I}_{Y^{n-1}, \tilde{\mathcal{P}}}(\sigma)\right)=h^{0}\left(q_{*} \mathcal{I}_{Y^{n-1}, \tilde{\mathcal{P}}}(\sigma)\right)=h^{0}\left(\mathcal{I}_{X^{n-1}, \mathcal{P}}{ }^{n+2}(\sigma)\right)$.

By our assumptions we can lift a section defining $V_{\sigma}$ to a nonzero element of $H^{0}\left(\mathcal{I}_{X^{n-1}, \mathcal{P}^{n+2}}(\sigma)\right)$. This contradiction proves $\tau \geq 0$.

This proves the first assertion of the theorem. As to the second, we need to prove that $\tau=0$. But $\tau$ being strictly positive would violate the usual uniqueness.

Remark 1.6. We used the surjectivity of the restriction maps only for $m=\sigma$.

Remark 1.7. The cases $\left(\mathcal{P}^{n+2}, L\right) \cong\left(\mathbb{P}^{n+2}, \mathcal{O}_{\mathbb{P}^{n+2}}(1)\right),\left(\mathcal{Q}^{4}, \mathcal{O}_{\mathcal{Q}^{4}}(1)\right)$ seem to be well known. See for example [1], [14], and of course [15]. However in the case of projective space it seems that the linear normality of $X$ was usually required; after Zak's theorem on tangencies linear normality is automatic, for a nonsingular $X$, unless $n=4$ and $X$ is the Veronese surface.

Example 1.8. The variety $\mathcal{P}^{n+2}$ can be, for example, a projective space, a nonsingular complete intersection or a Grassmannian; in all these cases $L$ is the hyperplane bundle for the natural embedding. But it can also be chosen to be a Fano variety, of index $r$, with $-K_{\mathcal{P}}=r L, L$ generated by global sections and $\operatorname{Pic}(\mathcal{P}) \simeq \mathbb{Z}$ (this is always the case if $r>n / 2$ ), some weighted complete intersections or, more generally, low degree branched coverings of projective spaces [12] or Grassmannians [11]. In the last batch of examples, $L$ does not need to be very ample.

The following gives a lifting criterion in any codimension; see [14]. Again linear normality is not required.

Corollary 1.9. Let $X^{\nu}$ be an integral subscheme of $\mathcal{P}^{n+2}$ of dimension $\nu, X^{\nu-1}$ the intersection of $X$ with a general member of $|L|, \sigma$ the postulation of $X^{\nu-1}$. Assume that $h^{0}\left(\mathcal{I}_{X^{\nu-1}}(\sigma)\right)=1$ and that $\rho_{\sigma}$ is surjective. Then $h^{0}\left(\mathcal{I}_{X^{\nu}}(\sigma)\right)=1$.

\section{Finiteness ON $\mathcal{Q}^{n}, n \geq 7$}

In this section we remark that, for a nonsingular variety of dimension $\nu \geq \frac{n+3}{2}$ in $\mathcal{Q}^{n}$ not of general type, the bound $d \leq 2 n^{n-\nu}$ holds. This gives the finiteness of the corresponding number of families.

We thank M. Schneider for pointing out to us that the result of this section could be proved along the lines of his paper [16]. 
Theorem 2.1. There are only finitely many components of the Hilbert scheme of $\mathcal{Q}^{n}$ corresponding to nonsingular subvarieties of dimension $\nu \geq \frac{n+3}{2}$ which are not of general type.

Proof. It suffices to bound the degree $d$ of any such $X$. The normal bundle $N$ of $X$ in $\mathcal{Q}^{n}$ is generated by global sections. The proposition of [16] is valid, on $X$, with "ample" replaced by "generated by global sections"; see [8], Example 12.1.7. It follows that

$$
c_{n-\nu}(N) \cdot c_{1}(N)^{2 \nu-n} \leq c_{1}(N)^{\nu} .
$$

By the self intersection formula for $X$ on $\mathcal{Q}^{n}$ and the structure of the cohomology ring of quadrics we have $c_{n-\nu}(N)=\frac{1}{2} d L^{n-\nu}$.

By [2], Theorem 2.3.11 we get that $\operatorname{Pic}(X) \simeq \mathbb{Z}[L]$, so that, if $X$ is not of general type, then $K_{X}=e L$, with $e \leq 0$. The adjunction formula gives $c_{1}(N)=(e+n) L$. By plugging into (5) we get

$$
\frac{1}{2} d^{2}(e+n)^{2 \nu-n} \leq(e+n)^{\nu} d
$$

which gives, after observing that $0 \leq-e \leq \operatorname{dim} X+1<n$, that

$$
d \leq 2(e+n)^{n-\nu} \leq 2 n^{n-\nu} .
$$

\section{3-FOLDS ON A HYPERSURFACE OF FIXED DEGREE}

In this section we generalize to the case of $\mathcal{Q}^{5}$ the main result of $\S 3$ of [4], which deals with bounds associated with nonsingular 3 -folds contained in a hypersurface of $\mathbb{P}^{5}$. For the analogous result on $\mathcal{Q}^{4}$ see [1], Proposition 6.7. However in both of the above references the result is proved under the assumption that " $d$ is big enough" with respect to the degree of the hypersurface. Of course this assumption is not a real restriction, since the residual cases are automatically taken care of by the fact that having a bounded degree bounds everything. However, it seems convenient to prove our statements without restrictions.

The importance of this bound is more or less theoretical: it can be used to assert the finiteness of special families of 3 -folds in $\mathcal{Q}^{5}$. One should not expect to make an effective use of them and get sharp results. The paper [7], which deals with surfaces in $\mathbb{P}^{4}$, is the original source of the main ideas used in [4], in $\S 6$ of [1], and in this section. The theoretical bound given there, for the degree of nonsingular surfaces not of general type in $\mathbb{P}^{4}$, is not an effective one. In the paper [3] an effective bound, $d \leq 105$, is proved using initial ideals.

Let $X$ be a 3 -fold of degree $d$ in $\mathcal{Q}^{5}$ contained in an integral hypersurface $V \in$ $\left|\mathcal{O}_{\mathcal{Q}^{5}}(\sigma)\right|, S$ a general hyperplane section of $X, C$ a general hyperplane section of $S$, and $g$ its genus. As a convention, when we write something like " + l.t. in $\sqrt{d}$," we mean that the coefficients of the lower terms depend only on $\sigma$.

Theorem 3.1. Let $X \subseteq V \subseteq \mathcal{Q}^{5}$ be as above. There is a degree eight polynomial $P_{\sigma}(\sqrt{d})$, depending only on $\sigma$ and with positive leading coefficient, such that

$$
-\chi\left(\mathcal{O}_{X}\right) \geq P_{\sigma}(\sqrt{d}) .
$$

Proof. Look at the following three exact sequences:

$$
0 \rightarrow \mathcal{O}_{\mathbb{P}^{6}}(t-2) \rightarrow \mathcal{O}_{\mathbb{P}^{6}}(t) \rightarrow \mathcal{O}_{\mathcal{Q}^{5}}(t) \rightarrow 0
$$




$$
\begin{aligned}
0 & \rightarrow \mathcal{O}_{\mathcal{Q}^{5}}(t-\sigma) \rightarrow \mathcal{O}_{\mathcal{Q}^{5}}(t) \rightarrow \mathcal{O}_{V}(t) \rightarrow 0 \\
0 & \rightarrow \mathcal{I}_{X, V}(t) \rightarrow \mathcal{O}_{V}(t) \rightarrow \mathcal{O}_{X}(t) \rightarrow 0
\end{aligned}
$$

One can use the first one to compute $\chi\left(\mathcal{O}_{\mathcal{Q}^{5}}(t)\right)$, the second one to compute

$$
\begin{aligned}
\chi\left(\mathcal{O}_{V}(t)\right)= & \frac{1}{12} \sigma t^{4}+\left(-\frac{1}{6} \sigma^{2}+\frac{5}{6} \sigma\right) t^{3}+\left(\frac{1}{6} \sigma^{3}-\frac{5}{4} \sigma^{2}+3 \sigma\right) t^{2} \\
& +\left(-\frac{1}{12} \sigma^{4}+\frac{5}{6} \sigma^{3}-3 \sigma^{2}+\frac{55}{12} \sigma\right) t \\
& +\frac{1}{60} \sigma^{5}-\frac{5}{24} \sigma^{4}+\sigma^{3}-\frac{55}{24} \sigma^{2}+\frac{149}{60} \sigma,
\end{aligned}
$$

and finally we use (4), $\mu:=\mu_{\sigma}=\frac{1}{2} d^{2}+\sigma(\sigma-3) d-2 \sigma(g-1)$ (cf. the notation fixed before Lemma 1.3), and the third short exact sequence to compute

$$
\begin{aligned}
\chi\left(\mathcal{I}_{X, V}(t)\right)= & \frac{1}{12} \sigma t^{4}+\frac{1}{6}[(5-\sigma) \sigma-d] t^{3} \\
& +\left[\frac{1}{6} \sigma^{3}-\frac{5}{4} \sigma^{2}+3 \sigma+\frac{1}{4 \sigma}\left(\frac{d^{2}}{2}+d \sigma(\sigma-3)-\mu\right)-\frac{d}{2}\right] t^{2} \\
& +\left[-\frac{1}{12} \sigma^{4}+\frac{5}{6} \sigma^{3}-3 \sigma^{2}+\frac{55}{12} \sigma-\frac{d}{3}\right. \\
& \left.\quad+\frac{1}{4 \sigma}\left(\frac{d^{2}}{2}+d \sigma(\sigma-3)-\mu\right)-\chi\left(\mathcal{O}_{S}\right)\right] t \\
& +\frac{1}{60} \sigma^{5}-\frac{5}{24} \sigma^{4}+\sigma^{3}-\frac{55}{24} \sigma^{2}+\frac{149}{60} \sigma-\chi\left(\mathcal{O}_{X}\right) \\
=: & Q(t)-\chi\left(\mathcal{O}_{X}\right) .
\end{aligned}
$$

It follows that

$$
-\chi\left(\mathcal{O}_{X}\right)=\chi\left(\mathcal{I}_{X, V}(t)\right)-Q(t)
$$

Define

$$
t_{1}:=\min \left\{t \in \mathbb{N} \mid \quad \delta:=2 \sigma t-d>0 \text { and } \frac{\delta^{2}}{2}-\mu-\delta \sigma(\sigma-3)>0\right\} ;
$$

by [1], page 89 ,

$$
\frac{d}{2 \sigma} \leq t_{1} \leq \frac{d}{2 \sigma}+\frac{\sqrt{2 d}}{2}+\sigma .
$$

By plugging $t_{1}$ into the above we get, using the above inequalities for $t_{1}$ and Lemma 1.3 ,

$-\chi\left(\mathcal{O}_{X}\right)=\chi\left(\mathcal{I}_{X, V}\left(t_{1}\right)\right)-Q\left(t_{1}\right) \geq \chi\left(I_{X, V}\left(t_{1}\right)\right)-\frac{1}{64 \sigma^{3}} d^{4}+\frac{1}{2 \sigma} \chi\left(\mathcal{O}_{S}\right) d+$ l.t. in $\sqrt{d} ;$

by [1], pages $88-89$, we also know that

$$
\chi\left(\mathcal{O}_{S}\right) \geq \frac{1}{24 \sigma^{2}} d^{3}+\text { l.t. in } \sqrt{d}
$$

so that

$$
-\chi\left(\mathcal{O}_{X}\right) \geq \chi\left(\mathcal{I}_{X, V}\left(t_{1}\right)\right)+\frac{1}{192 \sigma^{3}} d^{4}+\text { l.t. in } \sqrt{d} .
$$

To conclude it is enough to bound conveniently from below $\chi\left(\mathcal{I}_{X, V}\left(t_{1}\right)\right)=h^{0}-$ $h^{1}+h^{2}-h^{3}+h^{4}$. This, in turn, can be accomplished by bounding $h^{1}$ and $h^{3}$ from above. This is the content of the following technical lemmata. 
First we fix some notation. By taking general hyperplane sections we obtain the following diagram:

$$
\begin{aligned}
& \mathcal{Q}^{3} \subseteq \ldots \subseteq \mathcal{Q}^{n+1} \subseteq \mathcal{Q}^{n+2} \\
& \tilde{V}^{2} \rightarrow V^{2} \subseteq \ldots \subseteq V^{n} \subseteq V^{n+1}=V \\
& X^{1} \subseteq \ldots \subseteq X^{n-1} \subseteq X^{n}=X
\end{aligned}
$$

where $\tilde{V}^{2}$ is the normalization of $V^{2}$.

The following lemma is the analogue of [4], Lemma 3.3. It is proved in the same way using [1], Lemmata $6.10,6.11$ and 6.12 , instead of the lemmata from [7] quoted in [4].

Lemma 3.2. Let $X=X^{n}$ be a degree $d$ nonsingular $n$-dimensional subvariety of $\mathcal{Q}^{n+2}, n \geq 2$. Assume that $X$ is contained in an integral hypersurface $V=V^{n+1}$ $\in\left|\mathcal{O}_{\mathcal{Q}^{n+2}}(\sigma)\right|$. Define $t_{1}$ as above. Then there are constants $A_{1}, A_{2}$, depending only on $\sigma$, such that

$$
\sum_{\nu=t_{1}}^{\infty} h^{1}\left(\mathcal{I}_{X^{1}, \tilde{V}^{2}}(\nu)\right) \leq A_{1} \sqrt{d^{3}}+\text { l.t. in } \sqrt{d}
$$

and

$$
\sum_{\nu=0}^{t_{1}-1} h^{1}\left(\mathcal{I}_{X^{1}, \tilde{V}^{2}}(\nu)\right) \leq A_{2} \sqrt{d^{5}}+\text { l.t. in } \sqrt{d} \text {. }
$$

The next lemma merely generalizes Lemma 3.4 of [4]. It should be noted that the proof of it in [4] has a flaw since the argument does not work in the case $i=n+1$. However that case is not needed for our (and their) purposes. In any case we easily prove a bound also in that case.

Lemma 3.3. Let things be as in the previous lemma. Then

$$
h^{0}\left(\mathcal{I}_{X, V}\left(t_{1}\right)\right) \leq B_{0} \sqrt{d^{2 n-1}}+\text { l.t. in } \sqrt{d}
$$

for $i=1$,

$$
h^{1}\left(\mathcal{I}_{X, V}\left(t_{1}\right)\right) \leq B_{1} \sqrt{d^{2 n+1}}+\text { l.t. in } \sqrt{d}
$$

for $i=n-1, n$

$$
h^{i}\left(\mathcal{I}_{X, V}\left(t_{1}\right)\right) \leq B_{i} \sqrt{d^{2 i+1}}+\text { l.t. in } \sqrt{d} \text {, }
$$

and for $i=n+1$

$$
h^{n+1}\left(\mathcal{I}_{X, V}\left(t_{1}\right)\right) \leq B_{n+1} d^{n+1}+\text { l.t. in } \sqrt{d},
$$

where the $B_{i}$ 's are positive constants depending only on $\sigma$.

Proof. By looking at the sequences

$$
0 \rightarrow \mathcal{I}_{X^{i}, V^{i+1}}(k-1) \rightarrow \mathcal{I}_{X^{i}, V^{i+1}}(k) \rightarrow \mathcal{I}_{X^{i-1}, V^{i}}(k) \rightarrow 0,
$$


we deduce that

$$
\begin{aligned}
h^{0}\left(\mathcal{I}_{X, V}\left(t_{1}\right)\right) & \leq h^{0}\left(\mathcal{I}_{X^{n-1}, V^{n}}\left(t_{1}\right)\right)+h^{0}\left(\mathcal{I}_{X^{n}, V^{n+1}}\left(t_{1}-1\right)\right) \\
& \leq \sum_{k=1}^{t_{1}} h^{0}\left(\mathcal{I}_{X^{n-1}, V^{n}}(k)\right) \\
& \vdots \\
& \leq \sum_{k=1}^{t_{1}} \cdots \sum_{k=1}^{t_{1}} h^{0}\left(\mathcal{I}_{X^{1}, V^{2}}(k)\right) \quad((n-1) \text { summands }) \\
& \leq t_{1}^{n-2} \sum_{k=1}^{t_{1}} h^{0}\left(\mathcal{I}_{X^{1}, V^{2}}(k)\right) \leq t_{1}^{n-2} \sum_{k=1}^{t_{1}} h^{0}\left(\mathcal{I}_{X^{1}, \tilde{V}^{2}}(k)\right) \\
& \leq t_{1}^{n-2}\left(A_{0} \sqrt{d^{3}}+\text { l.t. in } \sqrt{d}\right),
\end{aligned}
$$

where the last inequality can be found in [1], Lemma 6.15 , and $A_{0}$ depends only on $\sigma$. Since $t_{1} \leq \frac{1}{2 \sigma} d+\frac{\sqrt{2 d}}{2}+\sigma$, we have bounded $h^{0}\left(\mathcal{I}_{X, V}\left(t_{1}\right)\right)$ as wanted.

To bound $h^{1}$ we argue as above.

$h^{1}\left(\mathcal{I}_{X, V}\left(t_{1}\right)\right) \leq \sum_{k=1}^{t_{1}} h^{1}\left(\mathcal{I}_{X^{n-1}, V^{n}}(k)\right)+h^{1}\left(\mathcal{I}_{X^{n}, V^{n+1}}\right)$, but this last dimension is zero as one can check by looking at the long cohomology sequences associated with the following two exact sequences:

$$
\begin{gathered}
0 \rightarrow \mathcal{I}_{X^{n}, \mathcal{Q}^{n+2}} \rightarrow \mathcal{O}_{\mathcal{Q}^{n+2}} \rightarrow \mathcal{O}_{X^{n}} \rightarrow 0 \\
0 \rightarrow \mathcal{O}_{\mathcal{Q}^{n+2}}(-\sigma) \rightarrow \mathcal{I}_{X^{n}, \mathcal{Q}^{n+2}} \rightarrow \mathcal{I}_{X^{n}, V^{n+1}} \rightarrow 0 .
\end{gathered}
$$

An easy induction argument, already seen before, using (6) allows us to infer that

$$
h^{1}\left(\mathcal{I}_{X, V}\left(t_{1}\right)\right) \leq t_{1}^{n-2} \sum_{k=1}^{t_{1}} h^{1}\left(\mathcal{I}_{X^{1}, V^{2}}(k)\right) .
$$

To obtain the desired bound on $h^{1}$ it is enough to prove that:

$$
\sum_{k=1}^{t_{1}} h^{1}\left(\mathcal{I}_{X^{1}, V^{2}}(k)\right) \leq F \sqrt{d^{5}}+\text { l.t. in } \sqrt{d},
$$

where $F$ is a constant depending only on $\sigma$.

This can be proved as follows. The idea is to couple the previous lemma with the cohomology sequences associated with the exact sequences

$$
0 \rightarrow \mathcal{I}_{X^{1}, V^{2}}(k) \rightarrow \mathcal{I}_{X^{1}, \tilde{V}^{2}}(k) \rightarrow Q(k) \rightarrow 0 .
$$

Clearly we have $h^{1}\left(\mathcal{I}_{X^{1}, V^{2}}(k)\right) \leq h^{1}\left(\mathcal{I}_{X^{1}, \tilde{V}^{2}}(k)\right)+h^{0}(Q(k)), \forall k$, so that, in view of Lemma 3.2, it is enough to prove the following

Claim. $h^{0}(Q(k)) \leq D(k+1), \forall k \geq 0$, where $D$ is a positive constant depending only on $\sigma$. In particular, $\sum_{k=0}^{t_{1}} h^{0}(Q(k)) \leq(1 / 2) D t_{1}^{2}+$ l.t. in $t_{1}$.

Proof. $Q$ is the structural sheaf of the non-normal locus of $V^{2}$ twisted by the ideal sheaf of $X^{1}$. By taking a general hyperplane section we get the following exact sequences:

$$
0 \rightarrow Q(k-1) \rightarrow Q(k) \rightarrow Q_{\Gamma}(k) \rightarrow 0,
$$

where $Q_{\Gamma}(k) \simeq Q_{\Gamma}$ is the structural sheaf of the singular locus of $\Gamma$, a general hyperplane section of $V^{2}$. As usual $h^{0}(Q(k)) \leq h^{0}(Q(k-1))+$ length $\left(Q_{\Gamma}\right)$, so that 
$h^{0}(Q(k)) \leq$ length $\left(Q_{\Gamma}\right)(k+1)+h^{0}(Q(-1))$. This length is bounded from above by a function of $\sigma$ only (that is, an irreducible curve of degree $2 \sigma$ on a two dimensional quadric cannot have too many singularities). As for $h^{0}(Q(-1))$, one has to exercise caution since the non-normal locus may be non-reduced. However by looking at the cohomology sequences associated with $(7)$ we see that $h^{1}\left(\mathcal{I}_{X^{1}, V^{2}}(-1)\right)=0$, so that $h^{0}(Q(-1))=0$, and the claim is proved.

Now we prove the bound for $i=n$.

We start by remarking that for $i=n-1, n, n+1$ and all $k \geq d-1$,

$$
h^{i}\left(\mathcal{I}_{X, V}(k)\right)=0 .
$$

If $X^{2}$ is nondegenerate then $\mathcal{I}_{X^{2}, \mathbb{P}^{5}}$ is $(d-2)$-regular (cf. [13]) in the sense of Castelnuovo-Mumford. By looking at the sequences

$$
0 \rightarrow \mathcal{I}_{X^{2}, \mathbb{P}^{5}}(-2+k) \rightarrow \mathcal{I}_{X^{2}, \mathbb{P}^{5}}(k) \rightarrow \mathcal{I}_{X^{2}, \mathcal{Q}^{4}}(k) \rightarrow 0,
$$

we deduce the vanishings, for $n=2$. An easy inductive argument (cf. [4], page $326)$ gives the desired vanishings.

If $X^{2} \subseteq \mathbb{P}^{5}$ were degenerate, then either $X^{2}=\mathbb{P}^{2}$, or $X^{2}$ would be a hypersurface of $\mathbb{P}^{3}$, or it would be a nondegenerate surface in $\mathbb{P}^{4}$. In any of these cases we apply the bound for the regularity of the ideal sheaves in [13] to obtain vanishings for the higher cohomology of $\mathcal{I}_{X^{2}, \mathbb{P}^{4}}$ which are easy to lift to the desired vanishings for $X^{2}$. Again the inductive procedure allows us to reach the desired conclusion.

We have the following chain of inequalities:

$$
\begin{aligned}
h^{n}\left(\mathcal{I}_{X^{n}, V^{n+1}}\left(t_{1}\right)\right) & \leq \sum_{k>t_{1}} h^{n-1}\left(\mathcal{I}_{X^{n-1}, V^{n+1}}(k)\right) \leq \sum_{k=1}^{d-4} h^{n-1}\left(\mathcal{I}_{X^{n-1}, V^{n+1}}(k)\right) \\
& \leq \ldots \leq \sum_{1}^{d-4} \ldots \sum_{1}^{d-4} \sum_{k=1}^{d-4} h^{1}\left(\mathcal{I}_{X^{1}, V^{2}}(k)\right) \\
& \leq(d-4)^{n-2} \sum_{k=1}^{d-4} h^{1}\left(\mathcal{I}_{X^{1}, V^{2}}(k)\right) \\
& \leq B_{n} \sqrt{d^{2 n+1}}+\text { l.t. in } \sqrt{d}
\end{aligned}
$$

where $B_{n}$ depends only on $\sigma$ and the last inequality follows from Lemma 3.2.

The case $i=n-1$ is analogous.

Finally, the bound for the case $i=n+1$ can be obtained as in the case $i=n$ except for the fact that we end up having to bound $h^{2}\left(\mathcal{I}_{X^{1}, V^{2}}(k)\right)$ for $k=1, \ldots$, $d-4$, and not $h^{1}$ :

$$
h^{n+1}\left(\mathcal{I}_{X, V}\left(t_{1}\right)\right) \leq d^{n-2}\left(\sum_{k=1}^{d-4} h^{2}\left(\mathcal{I}_{X, V}\left(t_{1}\right)\right) .\right.
$$

To bound this summand we look at the exact sequences

$$
0 \rightarrow \mathcal{I}_{X^{1}, V^{2}}(k) \rightarrow \mathcal{O}_{V^{2}}(k) \rightarrow \mathcal{O}_{X^{1}}(k) \rightarrow 0,
$$

and deduce that

$h^{2}\left(\mathcal{I}_{X^{1}, V^{2}}(k)\right) \leq h^{1}\left(\mathcal{O}_{X^{1}}(k)\right)+h^{2}\left(\mathcal{O}_{V^{2}}(k)\right)=h^{0}\left(\omega_{X^{1}}(-k)\right)+h^{0}\left(\mathcal{O}_{X^{1}}(-3+\sigma-k)\right)$

where the last equality stems from Serre duality. We are thus left with bounding the two $h^{0}$ above. The first one can be bounded using Proposition 1.1 on $h^{0}\left(\omega_{X^{1}}\right)=$ 
$g\left(X^{1}\right)$ : the worst upper bound is of the form $(1 / 4 \sigma) d^{2}+$ l.t. in $d$. As for the second $h^{0}$, its worst upper bound is of the form $(1 / 2) \sigma^{2}$. Adding up for $k=1, \ldots, d-4$, we get that the worst upper bound is $(1 / 4 \sigma) d^{3}+$ l.t. in $d$.

The following generalizes [4], Corollary 3.1.

Corollary 3.4. Let $\sigma$ be any positive integer. There are only finitely many components of the Hilbert scheme of $\mathcal{Q}^{5}$ corresponding to nonsingular 3 -folds in $\mathcal{Q}^{5}$ which are not of general type and are contained in some hypersurface of degree $\sigma$.

Proof. It is enough to bound from above the degree of such 3 -folds, since $\omega_{X}(-1)$ does not have sections $h^{0}\left(\omega_{X}\right) \leq h^{0}\left(\omega_{S}\right)$, where $S$ is any nonsingular hyperplane section of $X$. By the generalized Castelnuovo-type bounds of Harris (cf. [9]) we have

$$
h^{0}\left(\omega_{S}\right) \leq A d^{3}+\text { l.t. in } d,
$$

where $A$ is some constant; the Lefschetz hyperplane theorem, coupled with Proposition 1.1, ensures that

$$
h^{1}\left(\mathcal{O}_{X}\right)=h^{1}\left(\mathcal{O}_{S}\right) \leq h^{1}\left(\mathcal{O}_{C}\right) \leq \frac{1}{4 \sigma} d^{2}+\text { l.t. in } d .
$$

It follows that

$$
h^{0}\left(\omega_{S}\right) \geq h^{0}\left(\omega_{X}\right)=1+h^{2}\left(\mathcal{O}_{X}\right)-h^{1}\left(\mathcal{O}_{X}\right)-\chi\left(\mathcal{O}_{X}\right) \geq \frac{1}{192 \sigma^{3}} d^{4}+\text { l.t. in } \sqrt{d} .
$$

Comparing the two inequalities for $h^{0}\left(\omega_{S}\right)$, we conclude that $d$ is bounded.

\section{Finiteness for 3 -Folds not of General type in $\mathcal{Q}^{5}$}

Proposition 4.1. Let $X$ be a nonsingular 3 -fold in $\mathcal{Q}^{5}$ and $k$ a positive integer. Then

$$
\chi\left(\mathcal{O}_{S}\right) \leq \frac{2}{3} \frac{(g-1)^{2}}{d}-\frac{1}{24} d^{2}+\frac{5}{12} d .
$$

If $X$ is not of general type, then

$$
-\chi\left(\mathcal{O}_{X}\right) \leq \chi\left(\mathcal{O}_{S}\right)+\frac{1}{2} d^{2}-2 d+2
$$

if $d>2 k^{2}$ and $X$ is not of general type and not contained in any hypersurface of degree strictly less than $2 \cdot k$, then

$$
-\chi\left(\mathcal{O}_{X}\right) \leq \chi\left(\mathcal{O}_{S}\right)+\frac{1}{k} d^{2}+(k-4) d+2 .
$$

Proof. The first inequality stems from the generalized Hodge index theorem contained in [4]:

$$
d\left(K_{X}^{2} L\right) \leq\left(K_{X} L^{2}\right)^{2}
$$

we make explicit the left hand side using (2) and the right hand side using (1).

For the second one we look at

$$
0 \rightarrow K_{X}(-1) \rightarrow K_{X} \rightarrow K_{S}(-1) \rightarrow 0 .
$$

Since $X$ is not of general type $h^{0}\left(K_{X}(-1)\right)=0$, as otherwise $K_{X}$ would be big, i.e. a $\left|m K_{X}\right|$ would define a birational map. It follows that $h^{3}\left(\mathcal{O}_{X}\right)=h^{0}\left(K_{X}\right) \leq$ $h^{0}\left(K_{S}(-1)\right) \leq h^{0}\left(K_{S}\right)=h^{2}\left(\mathcal{O}_{S}\right)$.

We thus have

$$
-\chi\left(\mathcal{O}_{X}\right) \leq h^{1}\left(\mathcal{O}_{X}\right)+h^{3}\left(\mathcal{O}_{X}\right) \leq \chi\left(\mathcal{O}_{S}\right)+2 h^{1}\left(\mathcal{O}_{X}\right),
$$


where we have used the Lefschetz theorem on hyperplane sections to ensure that $h^{1}\left(\mathcal{O}_{X}\right)=h^{1}\left(\mathcal{O}_{S}\right)$. We have $h^{1}\left(\mathcal{O}_{S}(-1)\right)=0$ by Kodaira Vanishing, so that $h^{1}\left(\mathcal{O}_{X}\right)=h^{1}\left(\mathcal{O}_{S}\right) \leq g$.

If $C$ were contained in a $\mathbb{P}^{3}$ we would use Proposition 1.1 with $k=1$ to conclude. If $C$ were not in any surface of degree strictly less than $2 \cdot 2$ we would use Proposition 1.2 with $k=2$.

The third inequality is proved exactly as the second one by using Proposition 1.5 to ensure that a general curve section $C$ is not in any surface of the corresponding $\mathcal{Q}^{3}$ of degree strictly less than $2 \cdot k$, and Proposition 1.2 to bound the genus $g$ from above.

Proposition 4.2. Let $X$ be a nonsingular 3 -fold in $\mathcal{Q}^{5}$. Then

$$
60 \chi\left(\mathcal{O}_{S}\right) \geq \frac{3}{2} d^{2}-12 d+(d-48)(g-1)+24 \chi\left(\mathcal{O}_{X}\right)
$$

Proof. Denote by $s_{i}$ and $n_{i}$ the Segre and Chern classes respectively of the normal bundle $N$ of $X$ in $\mathcal{Q}^{5}$. Since $N$ is generated by global sections we have $s_{3} \geq 0$. Since $s_{3}=n_{1}^{3}-2 n_{1} n_{2}$ we get

$$
\begin{aligned}
0 & \leq\left(K_{X}+5 L\right)^{3}-2\left(K_{X}+5 L\right) \frac{1}{2} d L^{2} \\
& =K^{3}+15 K_{X}^{2} L+75 K_{X} L^{2}+125 d-d\left(K_{X}+5 L\right) L^{2} .
\end{aligned}
$$

We conclude by (3), (2) and (1).

Theorem 4.3. Let $n=4,5$ or $n \geq 7$. There are only finitely many components of the Hilbert scheme of $\mathcal{Q}^{n}$ corresponding to nonsingular $(n-2)$-folds not of general type.

Proof. By [1], $\S 6$, and Theorem 2.1 it is enough to consider the case $n=5$. It is enough to bound from above the degree $d$ of such 3 -folds.

Fix a positive integer $k$ and let $d$ be a positive integer such that $d>2 k^{2}$. Let $X$ be a degree $d 3$-fold in $\mathcal{Q}^{5}$ not lying on any hypersurface of $\mathcal{Q}^{5}$ of degree strictly less than $2 \cdot k$; by Proposition 1.5 , a general curve section of $X$ does not lie on any surface of the corresponding $\mathcal{Q}^{3}$ of degree strictly less than $2 \cdot k$.

We couple Proposition 4.2 and inequality (11) of Proposition 4.1:

$$
84 \chi\left(\mathcal{O}_{S}\right) \geq\left(\frac{3}{2}-\frac{24}{k}\right) d^{2}-(24 k-84) d-48+(d-48)(g-1) .
$$

We plug inequality (9) of Proposition 4.1 into the above and get:

$$
\frac{52}{d}(g-1)^{2}-\frac{21}{6} d^{2}+35 d \geq\left(\frac{3}{2}-\frac{24}{k}\right) d^{2}-(24 k-84) d-48+(d-48)(g-1) .
$$

A simple manipulation gives

$$
(g-1)\left[\frac{52}{d}(g-1)-d+48\right]+\left(\frac{24}{k}-5\right) d^{2}+(24 k-49) d+48 \geq 0 .
$$

Let us now first assume that $g>0$. The aim is to choose $k$ such that the coefficients $\alpha:=\left(\frac{52}{d}(g-1)-d+48\right)$ and $\beta:=\left(\frac{24}{k}-5\right)$ of $(g-1)$ and $d^{2}$, respectively, are negative. Once they are negative, since $k$ is fixed, the inequality (12) will force $d$ 
to be bounded from above. By Proposition 1.3 we get

$$
\begin{aligned}
\frac{52}{d}(g-1)-d+48 & \leq \frac{52}{d}\left[\frac{d^{2}}{2 k}+\frac{1}{2}(k-4) d\right]-d+48 \\
& =\left(\frac{26}{k}-1\right) d+26 k-56 .
\end{aligned}
$$

Let $k=27$; then $\alpha$ is negative for $d \gg 0$. For the same value of $k, \beta$ is negative as well. By the above we infer that $d$ is bounded from above if $g>0$ and $X$ is not in a hypersurface of degree strictly less than $2 \cdot 27$. We apply Corollary 3.4 to see that $d$ is bounded from above for 3 -folds $X$, not of general type, contained in hypersurfaces of degrees less than or equal to $2 \cdot 27$. This proves the theorem in the case $g>0$.

Assume that $g=0$. Then, by (12),

$$
\frac{52}{d}+d-48+\left(\frac{24}{k}-5\right) d^{2}+(24 k-49) d+48 \geq 0 .
$$

We argue as above with $k=5$.

\section{REFERENCES}

1. E. Arrondo, I. Sols, On congruences of lines in the projective space, Mém. Soc. Math. France (N.S.) No. 50 (1992) MR 93g:14040

2. M. Beltrametti and A.J. Sommese, The adjunction theory of complex projective varieties, vol. 16, de Gruyter, Berlin, 1995. MR 96f:14004

3. R. Braun, G. Floystad, "A bound for the degree of smooth surfaces in $\mathbb{P}^{4}$ not of general type," Compositio Math. 93 (1994), 211-229. MR 95g:14037

4. R. Braun, G. Ottaviani, M. Schneider, F.-O. Schreyer, "Boundedness of nongeneral type 3folds in $\mathbb{P}^{5}$," in Complex Analysis and Geometry, ed. by V. Ancona and A. Silva, Plenum Press, New York, 1993, pp. 311-338. MR 94b:14033

5. M.A.A. de Cataldo, Thesis, Notre Dame (1995).

6. M.A.A. de Cataldo, "The genus of curves on the three dimensional quadric," preprint (1994).

7. G. Ellingsrud, C. Peskine, "Sur les surfaces lisses de $\mathbb{P}^{4}$," Invent. Math. 95 (1989), 1-11. MR 89j: 14023

8. W. Fulton, Intersection Theory, Springer-Verlag, Berlin, (1984). MR 85k:14004

9. J. Harris, "A bound on the geometric genus of projective varieties," Ann. Scuola Norm. Sup. Pisa Cl. Sci. (4) 8 (1981), 35-68. MR 82h:14010

10. R. Hartshorne, Algebraic Geometry, Springer-Verlag, New York, (1978). MR 57:3116

11. M. Kim, "A Barth-Lefschetz type theorem for covering of Grassmannians," Reine Angew. Math. 470 (1996), 109-122. CMP 96:7

12. R. Lazarsfeld, "A Barth-type theorem for branched coverings of projective space," Math. Ann. 249 (1980), 153-162. MR 81g:14007

13. R. Lazarsfeld, "A sharp Castelnuovo bound for smooth surfaces," Duke Math. J. 55 (1987), 423-429. MR 89d:14007

14. E. Mezzetti, I. Raspanti, "A Laudal-type theorem for surfaces In $\mathbb{P}^{4}, "$ Rend. Sem. Mat. Univ. Pol. (a) Torino 48 (1990), 529-537; (b) E. Mezzetti, "Differential-geometric methods for the lifting problem and linear systems on plane curves", J. Differential Geometry 3 (1994), 375398. MR 95d:14049

15. L. Roth, "On the projective classification of surfaces," Proc. London Math. Soc. (2) 42 (1937), $142-170$.

16. M. Schneider, "Boundedness of low-codimensional submanifolds of projective space," Internat. J. Math. 3 (1992), 397-399. MR 93g:14057

Department of Mathematics, Washington University in St. Louis, Campus Box 1146, St. Louis, Missouri 63130-4899

E-mail address: mde@math.wustl.edu 